\title{
Diversité et sélection participative de variétés locales productives de banane plantain de Côte d'Ivoire
}

\author{
Thiémélé DEF1, Traoré $\mathbf{S}^{1}$, Aby $\mathbf{N}^{1}$, Gnonhouri $\mathbf{P}^{1}$, Yao N1 , Kobenan $\mathrm{K}^{1}$, Konan $\mathrm{E}^{2}$, Adiko $\mathrm{A}^{3}$, Zakra $\mathbf{N}^{3}$ \\ 1 Programme Plantain Banane Ananas, CNRA, Station Bimbresso 01 BP 1536 Abidjan 01, Côte d'Ivoire \\ 2 Direction Régionale d'Abidjan, CNRA, 08 BP 33 Abidjan 03, Côte d'Ivoire \\ ${ }^{3}$ Direction Générale, CNRA, 01 BP 1750 Abidjan 01, Côte d'Ivoire \\ Auteur correspondant : delessthiemele@gmail.com
}

Original submitted in on 20 March 2017. Published online at www.m.elewa.org on $30^{\text {th }}$ June 2017 https://dx.doi.org/10.4314/jab.v114i1.6

\section{RESUME}

Objectif : Parmi les principales cultures vivrières qui contribuent à la sécurité alimentaire en Côte d'Ivoire, la banane plantain occupe une place de choix. Cependant, la production reste toujours faible à cause de l'utilisation de variétés traditionnelles à faible rendement et aux mauvaises pratiques agricoles appliquées par les producteurs. Cette étude se propose d'identifier des variétés locales performantes pour accroire les rendements

Méthodologie et résultats : Pour apporter une solution à ces problèmes, plusieurs variétés locales de banane plantain ont été collectées dans les zones de production de bananiers en Côte d'Ivoire. Une diversité élevée de variétés de banane plantain a été observée à l'Est du pays. L'évaluation agronomique en station de recherche a permis de sélectionner de nombreux génotypes qui présentent des potentialités agronomiques intéressantes (haut rendement, tolérance aux maladies, précocité, etc.). Parmi ces variétés, 10 ont fait l'objet d'une sélection participative dans deux zones agro-écologiques différentes de la Côte d'Ivoire. Les résultats de cette évaluation ont permis de sélectionner 6 (CNRA-PLA-3, CNRA-PLA-4, CNRA-PLA-15, CNRA-PLA-7, CNRA-PLA-18 et CNRA-PLA-9) parmi les 10 variétés par les producteurs eux-mêmes. Ces variétés ont été choisies par les producteurs sur la base de leur rendement élevé, leur précocité et leur tolérance à la cercosporiose noire.

Conclusion et application des résultats : La présente étude a permis de mettre en évidence 6 variétés locales productives de banane plantain à la suite d'une sélection participative. Ces variétés peuvent être conseillées aux producteurs pour accroître ainsi les productions afin d'assurer la sécurité alimentaire des populations.

Mots clés : Banane plantain, rendement, tolérance, sélection participative, Côte d'Ivoire

\section{Diversity and participatory selection of high-yielding local plantain varieties in Côte d'Ivoire ABSTRACT}

Objective: Among the main food crops that contribute to food security in Côte d'Ivoire, plantain occupies a place of choice. However, the production is still low due to the use of traditional varieties with low yield and poor agricultural practices applied by the producers. This study aims to identify performant local plantain varieties to increase the yield.

Methods and Results: To provide a solution to these problems, several local varieties of plantain have been collected in plantains production areas in Côte d'Ivoire. A high diversity of plantain varieties has been observed in the east of the country. The agronomic evaluation in research station allowed to select many genotypes with 
interesting agronomic potential (high yield, tolerance to diseases, precocity, etc.). Among these varieties, 10 were used for participatory selection in two different agro-ecological areas in Côte d'Ivoire. The results of this evaluation allowed the producers to select 6 (CNRA-PLA-3, CNRA-PLA-4, CNRA-PLA-15, CNRA-PLA-7, CNRA-PLA-18 and CNRA-PLA-9) among the 10 varieties. These varieties have been chosen by the producers on the basis of their high yield, their precocity and their tolerance to black sigatoka.

Conclusion and application of results: The present study permitted to identify 6 performant local plantain varieties during a participatory selection. The varieties may be recommended to the producers in order to increase the productions and ensure the food security of the population.

Key words: Plantain, yield, tolerance, participatory selection, Cote d'Ivoire

\section{INTRODUCTION}

La banane plantain est un produit de grande consommation en Afrique Centrale et de l'Ouest (Tetang, 2013). Elle contribue de manière essentielle à la sécurité alimentaire, la création d'emplois, la diversification des revenus dans les zones rurales et urbaines, au produit intérieur brut (PIB) et, ce faisant, à la lutte contre la pauvreté (Nkendah, 2001). En Côte d'Ivoire, la banane plantain joue un rôle important non seulement dans l'alimentation des populations mais également dans les systèmes de culture. Avec une production annuelle estimée à 1,7 millions de tonnes (Anonyme, 2010), pour une consommation per capita variant entre 80 et $120 \mathrm{Kg}$, elle est la 3ème culture vivrière après l'igname et le manioc (Kouassi, 2006). Malgré son importance, la production reste toujours faible pour couvrir les besoins de la population en perpétuelle croissance. En effet, le secteur bananier plantain ivoirien est encore informel, caractérisé par des variétés traditionnelles peu productives (2 à $8 \mathrm{~kg} /$ régime) et les mauvaises pratiques agricoles par les

\section{MATÉRIEL ET MÉTHODES}

Collecte des variétés : Une prospection de collecte de variétés locales a été effectuée dans les zones de production de bananiers en Côte d'Ivoire. II s'agit des zones Est, Centre-Ouest, Sud-Ouest et Ouest où plusieurs villages ont été prospectés (Figure 1). Le climat dans les quatre zones est équatorial humide, avec quatre saisons dont deux humides et deux sèches. La collecte a été réalisée à travers une enquête de producteurs identifiés par l'Agence Nationale d'Appui au producteurs (Kouassi, 2006). Généralement, le bananier est utilisé en culture secondaire dans des systèmes traditionnels de production. Il est cultivé en association avec d'autres cultures vivrières (manioc, igname, riz, taro, etc.), des cultures de rente (café, cacao) ou des cultures fruitières (avocatier, agrumes etc.). Les systèmes en monoculture de bananier plantain sont plutôt rares (Temple et al., 2001; Traoré et al., 2009). L'utilisation des variétés à haut potentiel de rendement et des bonnes pratiques agricoles pourraient accroitre la productivité de la banane plantain en Côte d'Ivoire et permettre aux producteurs de tirer un meilleur revenu de leurs productions. Ainsi, l'identification de nouvelles variétés met en évidence l'exploitation et la valorisation de la biodiversité des ressources génétiques de bananier plantain.L'objectif de cette étude est de rechercher et caractériser de nouvelles variétés de bananier plantain à haut rendement pour contribuer à la réalisation de la sécurité alimentaire en Côte d'Ivoire.

Développement Rural (ANADER) de la zone. Les variétés ont été collectées au champ sur la base des noms locaux. Chaque prélèvement a été précédé d'un entretien avec le paysan qui autorise l'accès à son champ et fournit les échantillons. Ainsi, les informations relatives à la variété, à la zone géographique du lieu de la collecte, les données GPS, à l'identité de l'exploitant et de l'exploitation ont été renseignées. 


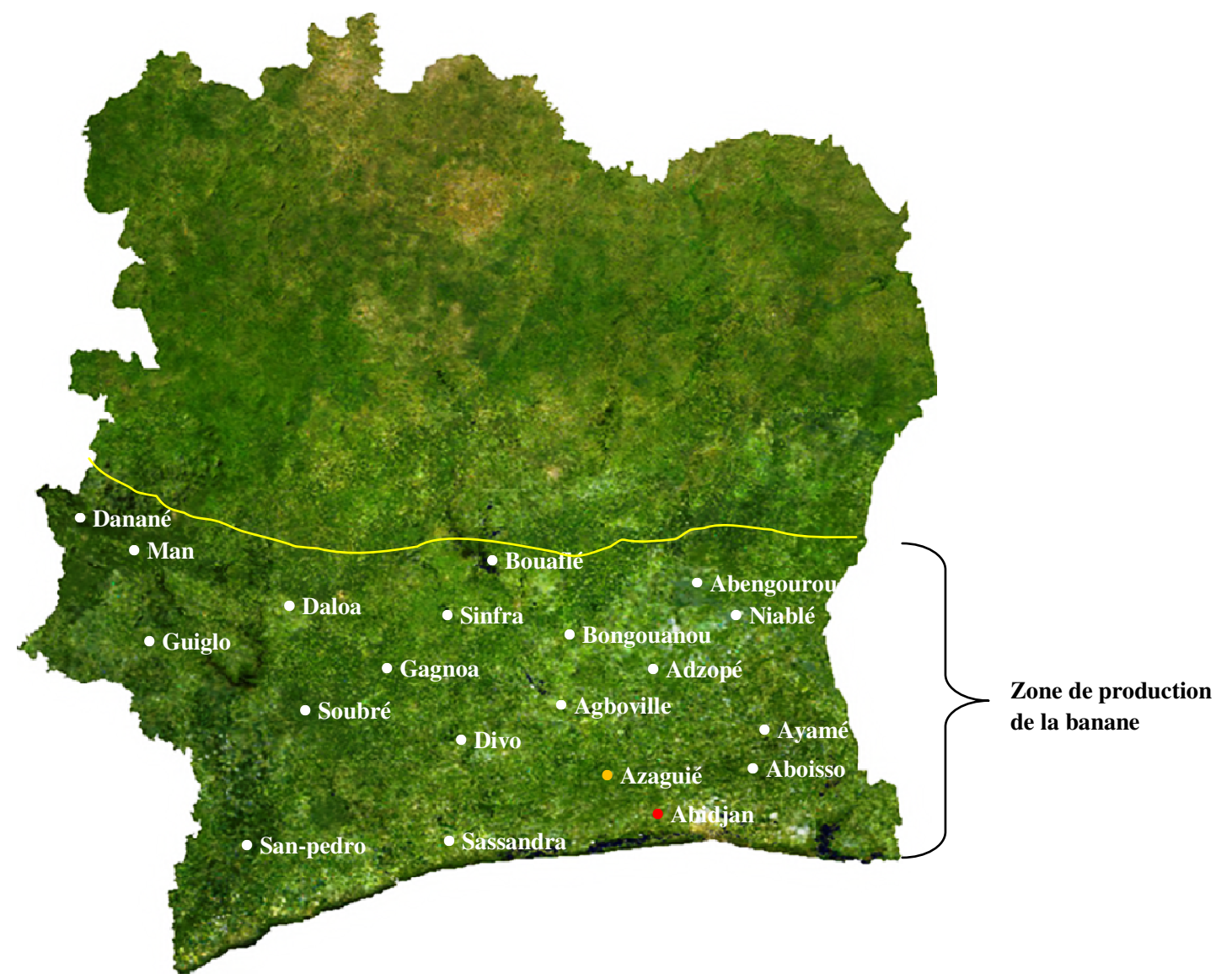

Figure 1 : Les différentes zones de prospection et de collecte des variétés locales de bananier plantain en Côte d'Ivoire.

Caractérisation agro-morphologique des variétés : Les variétés collectées ont été plantées à la station expérimentale du Centre National de Recherche Agronomique (CNRA) d'Azaguié $\left(5^{\circ} 18^{\prime}\right.$ de latitude Nord et $4^{\circ} 09^{\prime}$ de longitude Ouest). Les rejets collectés ont été parés pour enlever les racines et éliminer les galeries de charançons avant la plantation. Une densité de plantation de 1667 pieds/ha soit $2 \mathrm{~m} \times 3 \mathrm{~m}$ a été appliquée. Chaque variété a occupé une ligne de plantation. Les pratiques agricoles telles que la fertilisation, la gestion des mauvaises herbes, la lutte contre les nématodes et les charançons, le tuteurage ont été appliquées pour assurer un bon développement des plants. A la floraison et à la récolte, la caractérisation agro-morphologique des bananiers a été réalisée à l'aide d'observation en utilisant le descripteur IPGRI-INIBAP/CIRAD, 1996.

Sélection de variétés performantes : A la suite de la caractérisation agro-morphologique, des variétés locales performantes ont été présélectionnées. Un indice technique (IT) a été calculé sur la base de 3 critères choisis selon leur intérêt économique (Kouassi, 2006):

$\mathrm{IT}=(0,4) \mathrm{LD}+(0,3) \mathrm{GR}+(0,3) \mathrm{PR}$

$L D=$ longueur du doigt médian de la $2^{\text {ème }}$ main,

$\mathrm{GR}=$ Grade du doigt médian de la 2ème main,

$P R=$ poids du régime.

Sélection participative des variétés performantes : Les variétés ayant montré un IT élevé ont été présélectionnées pour un essai d'évaluation et de sélection participative sur 2 sites à l'intérieur du pays dans des conditions agro-écologiques différentes Katiola (Nord, zone marginale) et Bouaflé (Centre-Ouest, zone de production). Un essai composé de dix variétés locales et une variété sensible (ORISHELE) à la maladie de la cercosporiose noire a été mis en place. Un dispositif en blocs de Fisher avec trois répétitions et dix plants par répétition a été adopté.

Les paramètres agronomiques et de rendement suivants ont été enregistrés : la hauteur des plants depuis le sol jusqu'au point d'émergence de 
l'inflorescence au moment de la floraison, ainsi que la circonférence du pseudotronc à $1 \mathrm{~m}$ du sol, le nombre de feuilles vivantes à la floraison, le poids du régime, le nombre de mains et de doigts par régime et la longueur du doigt mesurée sur le doigt extérieur du milieu de la deuxième main. La réaction des variétés à la

\section{RESULTATS}

Variétés collectées : Au total, ce sont 102 variétés de bananier plantain qui ont été collectées dans les différentes zones prospectées (Tableau 1) dont 51 à l'Est, 24 au Centre-Ouest, 16 au Sud-Ouest et 11 à l'Ouest. Un nombre total de 623 rejets ont été prélevés avec une moyenne de 6 rejets par variétés. La biodiversité des cercosporiose noire a été évaluée en enregistrant la plus jeune feuille nécrosée (PJFN) à la floraison.

Analyse statistique: Tous ces paramètres ont été soumis à une analyse de variance en utilisant le logiciel STATISTICA version 6. Le test de Newman-Keuls a été utilisé pour séparer les moyennes au seuil de $5 \%$.

ressources génétiques de plantain a été plus riche à l'Est du pays avec $50 \%$ des variétés collectées. Cette diversité baisse de l'Est vers l'Ouest de la Côte d'Ivoire par la réduction du nombre de variétés identifiés (Tableau 1).

Tableau 1 : Nombre de variétés de bananier plantain collectées dans les différentes zones en Côte d'Ivoire

\begin{tabular}{l|c|c|c|c}
\hline Zone & Est & Centre-ouest & Sud-ouest & Ouest \\
\hline Localités prospectées & $\begin{array}{c}\text { Abengourou, Niablé, Aboisso, } \\
\text { Ayamé, Adzopé Bongouanou } \\
\text { Agboville }\end{array}$ & $\begin{array}{c}\text { Bouaflé, Sinfra, } \\
\text { Gagnoa, Divo } \\
\text { Daloa }\end{array}$ & $\begin{array}{c}\text { Soubré, San- } \\
\text { pedro, } \\
\text { Sassandra }\end{array}$ & $\begin{array}{c}\text { Man, Danané, } \\
\text { Guiglo }\end{array}$ \\
\hline $\begin{array}{l}\text { Nombre de variétés } \\
\text { collectées }\end{array}$ & 51 & 24 & 16 & 11 \\
\hline $\begin{array}{l}\text { Nombre total de rejets } \\
\text { collectés }\end{array}$ & 306 & 144 & 96 & 77 \\
\hline
\end{tabular}

Caractéristiques agro-morphologique des variétés : La caractérisation agro-morphologique des variétés collectées a permis de mettre en évidence les trois types de plantain les plus cultivés dans le monde (Figure 2). II s'agit des types French, Vrai corne et Faux corne. Un quatrième type a été également mis en évidence, le type Batard qui est intermédiaire entre le Faux corne et French (Figure 2). Le type Batard a été identifié uniquement dans les zones Est et Centre-Ouest. Les types Faux corne ont été les plus nombreux suivit respectivement des types French, Vrai corne et intermédiaire quelque soit la zone de collecte (Figure 3). La caractérisation agromorphologique a permis également de mettre en évidence des doublons dans les échantillons collectés. Ainsi 14 variétés (8 French et 6 Faux corne) ont été identifiées comme des doublons réduisant le nombre de la collection à 88 variétés. 
Thiémélé et al., J. Appl. Biosci. 2017 Diversité et sélection participative de variétés locales productives de banane plantain de Côte d'Ivoire

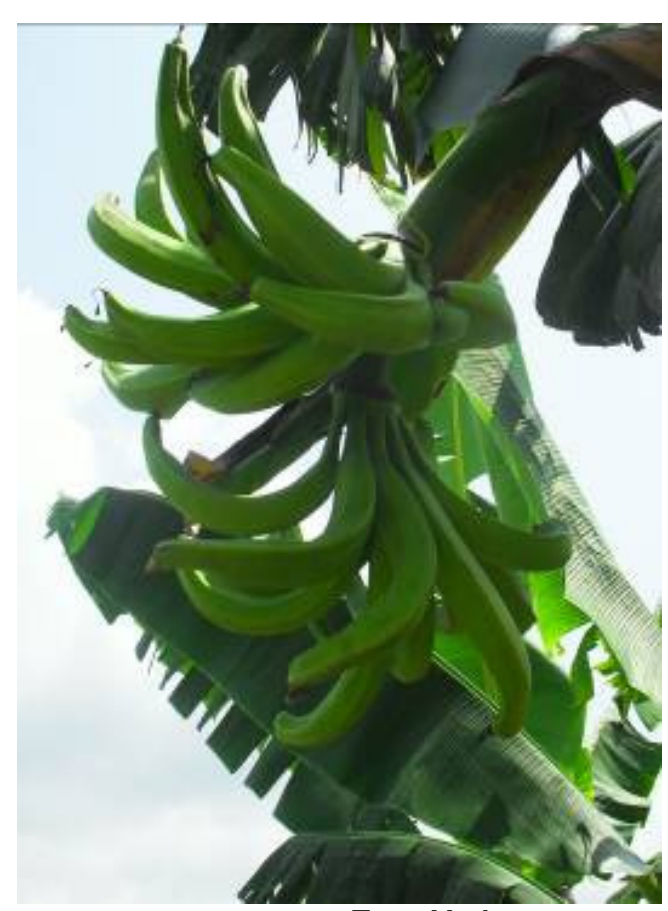

Type Vrai corne

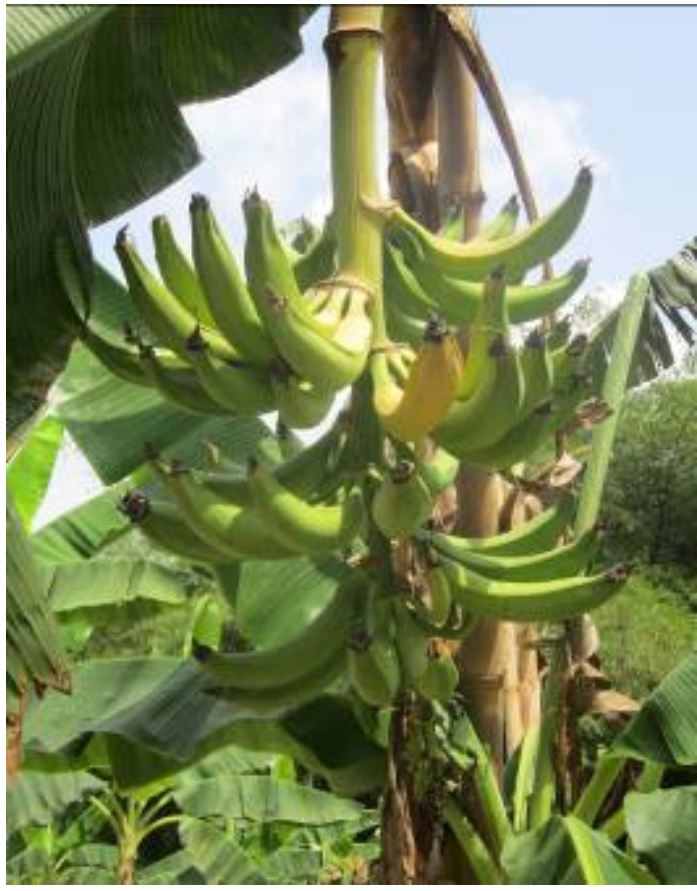

Type Faux corne

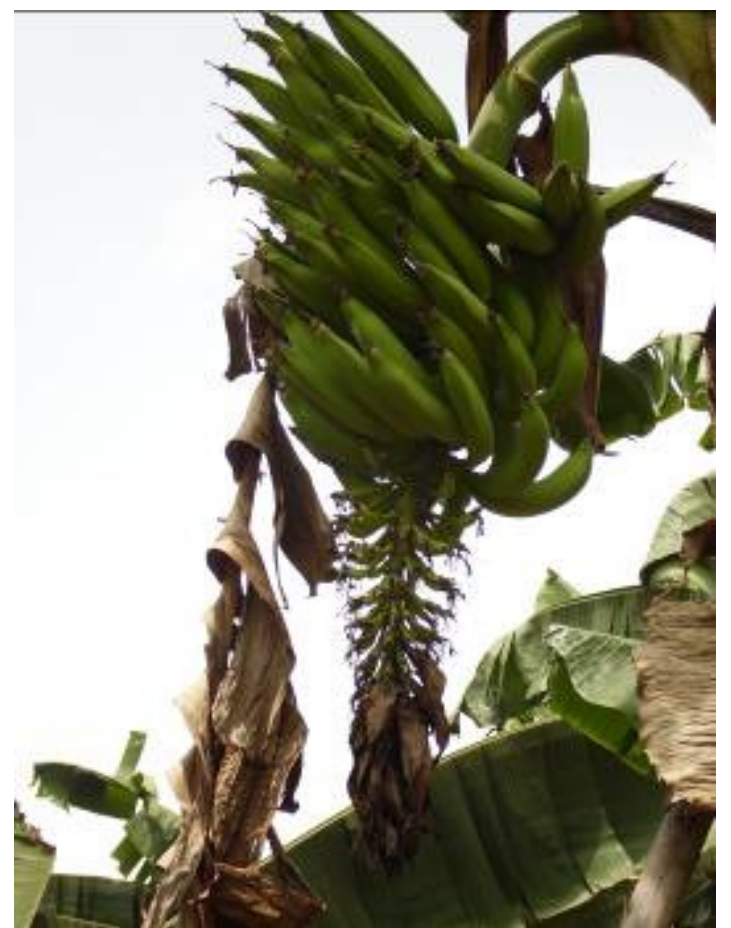

Type intermédiaire

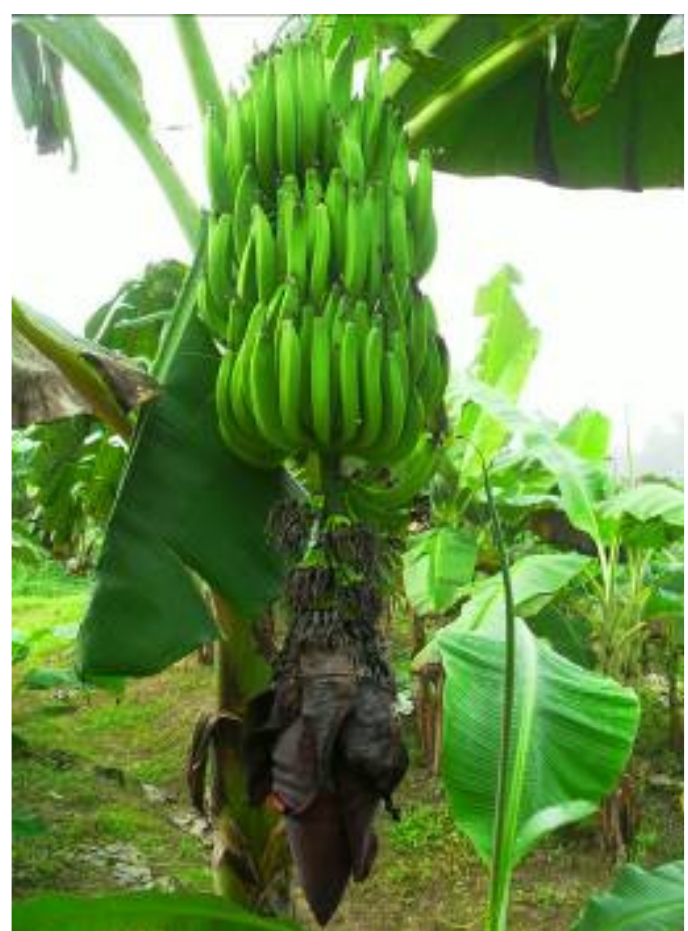

Type French

Figure 2 : Les différents types de variétés de bananier plantain rencontrés en Côte d'Ivoire 


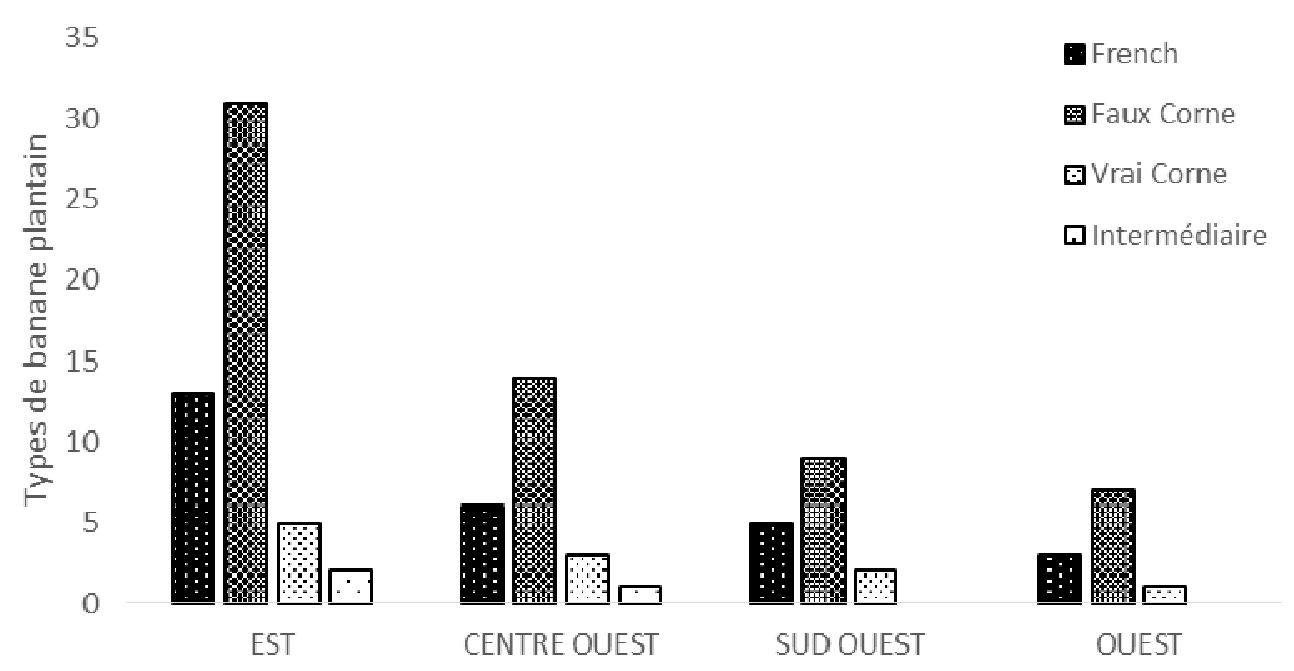

Figure 3 : Les différents types de variétés de bananier plantain identifiés dans les différentes zones en Côte d'Ivoire

Performances agronomiques et sélection des variétés élites dans les zones agro-écologiques : Parmi les 88 variétés évaluées au cours de la caractérisation agromorphologique à Azaguié, les 10 premières qui ont montré les indices techniques les plus élevés (IT $\geq 20$ ) ont été présélectionnées pour faire l'objet d'une évaluation et d'une sélection participative à Katiola et à Bouaflé (Tableau 2). Le tableau 3 présente les caractéristiques agronomiques des variétés évaluées dans le cadre de la sélection participative. Aucune différence significative $(P \leq 0,05)$ n'a été mise en évidence entre les cycles de production. Les variétés ont eu un cycle de production court variant de $331 \pm 0,97$ jours (cv. CNRA-PLA-11) à 360 $\pm 0,97$ jours (CNRA-PLA-4). Toutes les variétés testées ont eu une hauteur de plant supérieure à $3 \mathrm{~m}$. La variété CNRA-PLA-4 a été la plus haute des variétés avec $380 \pm 0,83 \mathrm{~cm}$ de hauteur. Des différences significatives $(P \leq 0,05)$ ont été également observées entre les variétés en ce qui concerne la circonférence du pseudtronc, le nombre de feuilles vivantes à la floraison, le poids du régime, la longueur du doigt et le nombre de doigts par régime. Les variétés CNRA-PLA-3, CNRA-PLA-4, CNRA-PLA-15, CNRA-PLA7, CNRA-PLA-18 et CNRA-PLA-9 ont montré les valeurs les plus élevées des caractéristiques agronomiques évaluées. En ce qui concerne le poids des régimes, il a

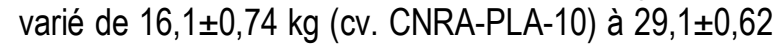
$\mathrm{kg}$ (CNRA-PLA-7). Les poids des régimes des variétés CNRA-PLA-3, CNRA-PLA-4, CNRA-PLA-15, CNRA-PLA7, CNRA-PLA-18 et CNRA-PLA-9 ont été les plus élevés. La variété CNRA-PLA-7 ayant produit le poids de régime le plus élevé se caractérise également par un grand nombre de mains $(9 \pm 0,77)$ et de doigts $(120 \pm 0,77)$ par régime. Les variétés type French ont eu les doigts assez courts $(27$ à $28 \mathrm{~cm})$ que les types Faux corne (30 à 35 $\mathrm{cm})$. Les performances des variétés ont été stables dans les différentes localités, tant en ce qui concerne le rendement que les caractéristiques de la croissance. La réaction à la cercosporiose noire a été évaluée en enregistrant la plus jeune feuille nécrosée (PJFN) à la floraison des plants. Toutes les variétés ont exprimé les symptômes de la maladie dans les deux localités (Tableau 4). Cependant, le rang de la plus jeune feuille nécrosée a été plus élevée (rand 6) que le témoin sensible (ORISHELE) (rang 4). Le rang a été également plus important dans la localité de Katiola (rang 8) que dans celle de Bouaflé (rang 6). Les variétés peuvent être considérés comme moins sensibles à la cercosporiose noire à Bouaflé et partiellement résistantes à Katiola. 
Tableau 2 : Indices techniques des 20 premières variétés de bananier plantain évaluées à Azaguié

\begin{tabular}{l|l|l|l|l|l|l}
\hline $\mathbf{N}^{\circ}$ & Variétés & $\begin{array}{l}\text { Type de } \\
\text { plantain }\end{array}$ & $\begin{array}{l}\text { Longueur du } \\
\text { doigt } \mathbf{( c m})\end{array}$ & $\begin{array}{l}\text { Poids du } \\
\text { régime } \mathbf{( k g})\end{array}$ & $\begin{array}{l}\text { Grade du } \\
\text { doigt } \mathbf{( c m})\end{array}$ & $\begin{array}{l}\text { Indice } \\
\text { technique (IT) }\end{array}$ \\
\hline 1 & CNRA-PLA-4 & Intermédiaire & 34 & 29.5 & 8.62 & 25.04 \\
\hline 2 & CNRA-PLA-15 & Faux Corne & 36 & 27.6 & 7.53 & 24.94 \\
\hline 3 & CNRA-PLA-3 & Faux Corne & 33 & 28.1 & 7.07 & 23.75 \\
\hline 4 & CNRA-PLA-7 & French & 28 & 33.6 & 6.52 & 23.24 \\
\hline 5 & CNRA-PLA-18 & French & 28 & 29.6 & 6.53 & 22.04 \\
\hline 6 & CNRA-PLA-9 & French & 28 & 28.2 & 6.55 & 21.63 \\
\hline 7 & CNRA-PLA-11 & Faux Corne & 32 & 18.7 & 7.28 & 20.59 \\
\hline 8 & CNRA-PLA-6 & Faux Corne & 31 & 19.9 & 6.93 & 20.45 \\
\hline 9 & CNRA-PLA-12 & French & 31 & 18.8 & 6.99 & 20.14 \\
\hline 10 & CNRA-PLA-10 & Faux Corne & 31 & 18.1 & 7.32 & 20.03 \\
\hline 11 & CNRA-PLA-2 & Faux Corne & 31 & 15.5 & 5.89 & 18.82 \\
\hline 12 & CNRA-PLA-14 & Faux Corne & 31 & 16 & 5.01 & 18.70 \\
\hline 13 & CNRA-PLA-30 & Faux Corne & 31 & 15.2 & 5.66 & 18.66 \\
\hline 14 & CNRA-PLA-3 & Faux Corne & 30 & 16.1 & 5.33 & 18.43 \\
\hline 15 & CNRA-PLA-25 & Faux Corne & 29 & 16.7 & 5.96 & 18.40 \\
\hline 16 & CNRA-PLA-1 & Faux Corne & 30 & 14.3 & 5.63 & 17.98 \\
\hline 17 & CNRA-PLA-41 & French & 28 & 16.8 & 4.69 & 17.65 \\
\hline 18 & CNRA-PLA-17 & French & 26 & 16.9 & 4.33 & 16.77 \\
\hline 19 & CNRA-PLA-21 & French & 25 & 16.3 & 4.99 & 16.39 \\
\hline 20 & CNRA-PLA-19 & French & 25 & 16 & 5.01 & 16.30 \\
\hline
\end{tabular}


Tableau 3 : Caractéristiques agronomiques des variétés locales évaluées au cours de la sélection participative.

\begin{tabular}{|c|c|c|c|c|c|c|c|c|c|}
\hline Variétés & $\begin{array}{l}\text { Type de } \\
\text { plantain }\end{array}$ & $\begin{array}{l}\text { Hauteur du } \\
\text { pseudotronc } \\
\text { (cm) }\end{array}$ & $\begin{array}{l}\text { Circonférence du } \\
\text { pseudotronc }(\mathrm{cm})\end{array}$ & $\begin{array}{l}\text { Longueur } \\
\text { du doigt } \\
\text { (cm) }\end{array}$ & $\begin{array}{c}\text { Nombre de } \\
\text { feuilles } \\
\text { vivantes }\end{array}$ & $\begin{array}{l}\text { Poids du } \\
\text { régime (kg) }\end{array}$ & $\begin{array}{c}\text { Nombre } \\
\text { de mains } \\
\text { par } \\
\text { régime }\end{array}$ & $\begin{array}{l}\text { Nombre } \\
\text { de doigts } \\
\text { par } \\
\text { régime }\end{array}$ & $\begin{array}{c}\text { Cycle de } \\
\text { production : } \\
\text { Intervalle } \\
\text { plantation- } \\
\text { récolte (jours) }\end{array}$ \\
\hline $\begin{array}{l}\text { CNRA- } \\
\text { PLA-3 }\end{array}$ & Faux Corne & $320^{b} \pm 0,72$ & $45^{b} \pm 0,97$ & $30^{b} \pm 0,51$ & $8^{b} \pm 0,58$ & $25,8^{b} \pm 0,76$ & $7^{\mathrm{a}} \pm 0,58$ & $97^{b} \pm 1,01$ & $337^{a} \pm 1,17$ \\
\hline $\begin{array}{l}\text { CNRA- } \\
\text { PLA-4 }\end{array}$ & Intermédiaire & $380^{a} \pm 0,83$ & $42^{b} \pm 0,77$ & $33^{b} \pm 1,75$ & $9^{b} \pm 0,50$ & $26,1^{\mathrm{b}} \pm 0,74$ & $7^{\mathrm{a}} \pm 0,58$ & $99^{b} \pm 0,72$ & $360^{a} \pm 0,97$ \\
\hline $\begin{array}{l}\text { CNRA- } \\
\text { PLA-15 } \\
\end{array}$ & Faux Corne & $330^{b} \pm 0,72$ & $44^{b} \pm 0,72$ & $35^{b} \pm 1,05$ & $7^{a b} \pm 0,58$ & $25,3^{b} \pm 0,42$ & $7^{\mathrm{a}} \pm 0,58$ & $80^{b} \pm 0,58$ & $340^{a} \pm 0,83$ \\
\hline $\begin{array}{l}\text { CNRA- } \\
\text { PLA-6 }\end{array}$ & Faux Corne & $332^{b} \pm 1,17$ & $35^{\mathrm{a}} \pm 0,72$ & $29^{a b} \pm 1,20$ & $6^{a} \pm 0,50$ & $16,1^{\mathrm{a}} \pm 0,80$ & $6^{\mathrm{a}} \pm 0,50$ & $56^{\mathrm{a}} \pm 0,77$ & $343^{a} \pm 0,97$ \\
\hline $\begin{array}{l}\text { CNRA- } \\
\text { PLA-7 }\end{array}$ & French & $347^{a b} \pm 1,09$ & $43^{b} \pm 0,77$ & $27^{\mathrm{a}} \pm 1,37$ & $8^{b} \pm 0,58$ & $29,1^{b} \pm 0,62$ & $9 \mathrm{a} \pm 0,77$ & $120^{\mathrm{b}} \pm 0,77$ & $344^{a} \pm 1,20$ \\
\hline $\begin{array}{l}\text { CNRA- } \\
\text { PLA-18 }\end{array}$ & French & $350^{\mathrm{ab}} \pm 0,72$ & $41^{b} \pm 1,05$ & $27^{\mathrm{a}} \pm 1,37$ & $8^{b} \pm 0,58$ & $27,6^{b} \pm 1,06$ & $8^{a} \pm 0,77$ & $103^{b} \pm 0,88$ & $346^{a} \pm 0,72$ \\
\hline $\begin{array}{l}\text { CNRA- } \\
\text { PLA-9 } \\
\end{array}$ & French & $315^{b} \pm 0,92$ & $42^{b} \pm 0,72$ & $27^{\mathrm{a}} \pm 1,37$ & $8^{b} \pm 0,58$ & $26,2^{b} \pm 1,04$ & $8^{a} \pm 0,77$ & $100^{b} \pm 0,77$ & $345^{a} \pm 1,09$ \\
\hline $\begin{array}{l}\text { CNRA- } \\
\text { PLA-10 }\end{array}$ & Faux Corne & $319^{b} \pm 0,72$ & $38^{a} \pm 1,09$ & $30^{b} \pm 0,51$ & $5^{\mathrm{a}} \pm 0,51$ & $16,1^{\mathrm{a}} \pm 0,74$ & $6^{a} \pm 0,51$ & $47^{\mathrm{a}} \pm 0,83$ & $337^{a} \pm 1,01$ \\
\hline $\begin{array}{l}\text { CNRA- } \\
\text { PLA-11 }\end{array}$ & Faux Corne & $329^{b} \pm 1,13$ & $36^{a} \pm 1,46$ & $30^{b} \pm 0,51$ & $6^{a} \pm 0,51$ & $16,5^{\mathrm{a}} \pm 0,67$ & $6^{\mathrm{a}} \pm 0,51$ & $45^{\mathrm{a}} \pm 0,65$ & $331^{a} \pm 0,97$ \\
\hline $\begin{array}{l}\text { CNRA- } \\
\text { PLA-12 }\end{array}$ & French & $340^{a b} \pm 0,92$ & $37^{a} \pm 0,97$ & $28^{a b} \pm 1,27$ & $6^{a} \pm 0,51$ & $16,8^{a} \pm 0,64$ & $7^{a} \pm 0,58$ & $57^{a} \pm 0,92$ & $335^{a} \pm 1,57$ \\
\hline CV (\%) & & 5,1 & 4,1 & 7,2 & 3,5 & 9,6 & 5,6 & 10,1 & 4,9 \\
\hline
\end{tabular}

Dans une même colonne, les moyennes suivies de la même lettre ne présentent pas différence significative selon le test de Newman-Keuls au seuil 5 \%. 

banane plantain de Côte d'Ivoire

Tableau 4 : Réaction des variétés à la cercosporiose noire, mesurée par la plus jeune feuille nécrosée (PJFN) dans les différentes zones agro-écologiques.

\begin{tabular}{l|l|l}
\hline Variétés & Bouaflé & Katiola \\
\hline CNRA-PLA-3 & $6,8^{\mathrm{b}} \pm 0,40$ & $8,4^{\mathrm{b}} \pm 0,43$ \\
\hline CNRA-PLA-4 & $6,5^{\mathrm{b}} \pm 0,44$ & $8,4^{\mathrm{b}} \pm 0,43$ \\
\hline CNRA-PLA-15 & $6,4^{\mathrm{b}} \pm 0,44$ & $8,3^{\mathrm{b}} \pm 0,51$ \\
\hline CNRA-PLA-6 & $6,6^{\mathrm{b}} \pm 0,43$ & $8,0^{\mathrm{b}} \pm 0,41$ \\
\hline CNRA-PLA-7 & $6,8^{\mathrm{b}} \pm 0,40$ & $8,6^{\mathrm{b}} \pm 0,43$ \\
\hline CNRA-PLA-18 & $6,8^{\mathrm{b}} \pm 0,40$ & $8,6^{\mathrm{b}} \pm 0,43$ \\
\hline CNRA-PLA-9 & $6,4^{\mathrm{b}} \pm 0,32$ & $8,7^{\mathrm{b}} \pm 0,30$ \\
\hline CNRA-PLA-10 & $6,0^{\mathrm{b}} \pm 0,41$ & $8,0^{\mathrm{b}} \pm 0,41$ \\
\hline CNRA-PLA-11 & $6,1^{\mathrm{b}} \pm 0,46$ & $8,0^{\mathrm{b}} \pm 0,41$ \\
\hline CNRA-PLA-12 & $6,0^{\mathrm{b}} \pm 0,41$ & $8,1^{\mathrm{b}} \pm 0,35$ \\
\hline Orishele (Témoin sensible) & $4,1^{\mathrm{a}} \pm 0,35$ & $6,3^{\mathrm{a}} \pm 0,42$ \\
\hline CV (\%) & 3,1 & 4,8 \\
\hline
\end{tabular}

\section{DISCUSSION}

Une forte diversité variétale a été observée lors de la prospection et la collecte de variétés locales de bananier plantain dans les zones de production en Côte d'Ivoire. Bien que le bananier soit originaire de l'Asie du Sud-est, où il est retrouvé de l'Inde à la Polynésie (Frison et al., 1998), une diversité génétique considérable a été mise en évidence en Afrique de l'Ouest, dans le Golfe de Guinée faisant de cette région une zone secondaire de diversification des bananiers plantain. De ce fait, les producteurs sont en contact avec plusieurs variétés qu'ils cultivent en mélange. Cette diversité a été plus élevée à l'Est du pays, ce qui pourrait s'expliquer par le fait que la banane plantain est l'aliment de base des communautés de cette région. Elle diminue ainsi de l'Est vers l'Ouest. En outre, les paysans utilisent traditionnellement le bananier plantain pour créer l'ombrage nécessaire au bon développement des jeunes cacaoyers et caféiers. La dynamique spatio-temporelle de la production de banane plantain est liée à celle du binôme café/cacao. La zone far de production de cacao, appelée 'boucle du cacao' était située à l'Est de la Côte d'Ivoire jusqu'à la fin des années 1980. Au début des années 1990, avec la diminution des forêts vierges, la boucle du cacao s'est déplacée au Centre-Ouest (région de Gagnoa). Aujourd'hui, elle évolue vers l'Ouest (région de Duékoué, Man) toujours à la conquête de nouvelles forêts (Kouassi, 2006). Utilisées habituellement en pool génétique, les ressources génétiques du plantain sont distribuées dans l'espace et dans le temps en suivant la dynamique de la boucle du cacao. Ainsi, les communautés attachées à la culture du café et du cacao ont contribué à la dissémination des ressources génétiques de l'Est vers l'Ouest en conservant un pool réduit de variétés en fonction de leur préférence. La plupart des variétés identifiées et collectées ont eu des appellations différentes en fonction des ethnies du producteur qui met disposition le matériel végétal. Plusieurs critères de description variétale sont utilisés par les producteurs pour classifier les variétés entre elles. II s'agit de la couleur et la taille du régime, la grosseur, la longueur et l'orientation des doigts, l'existence ou non du bourgeon mâle, la couleur du pseudotronc, ainsi que le goût et la tendreté de la pulpe de plantain. 


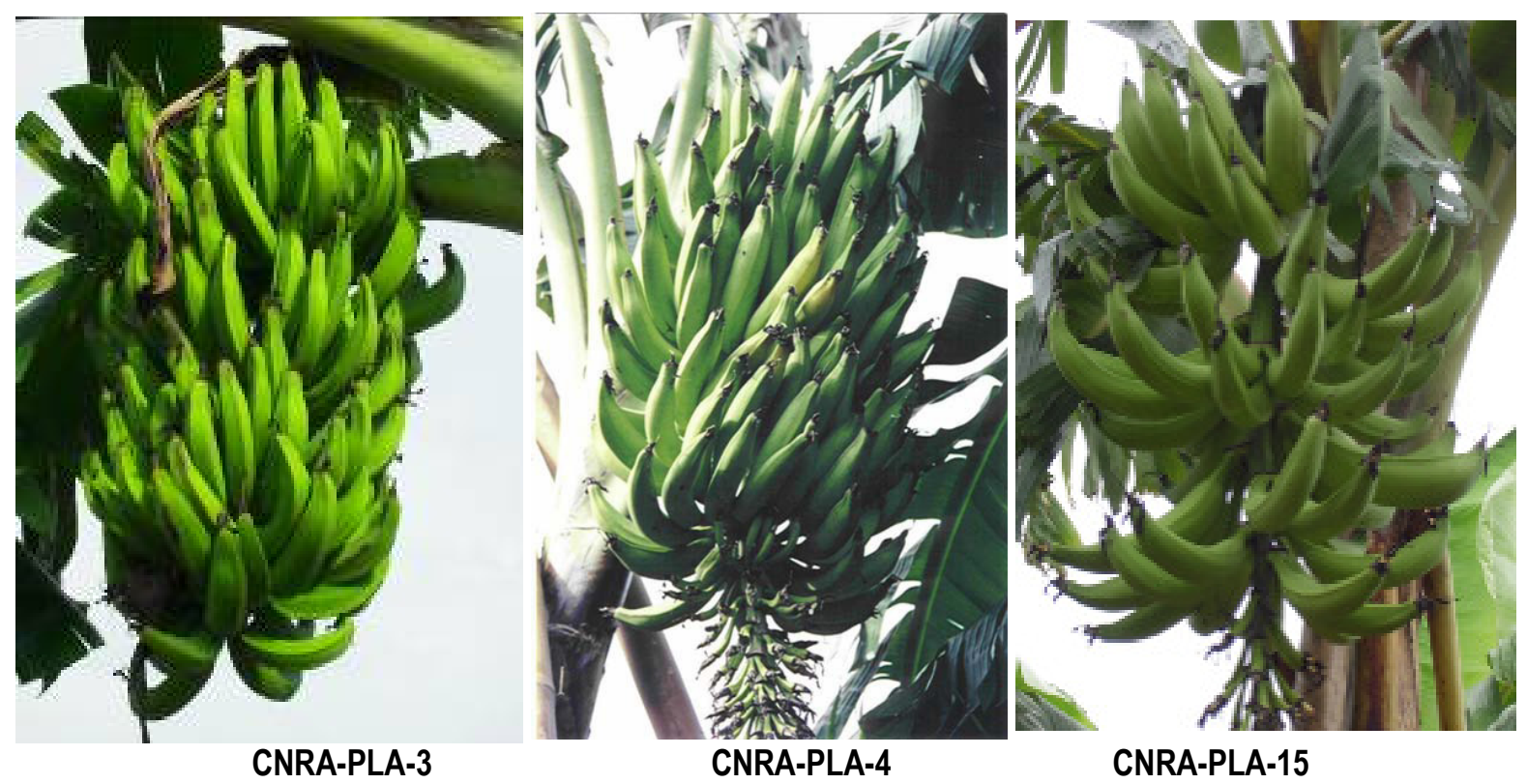

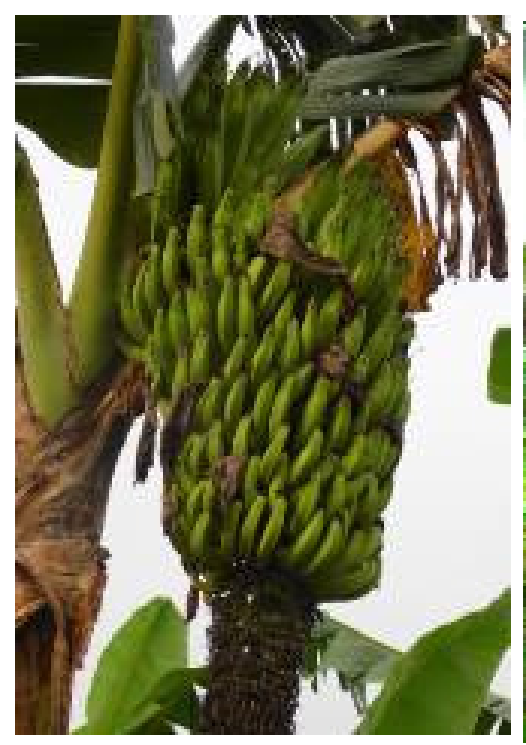

CNRA-PLA-7

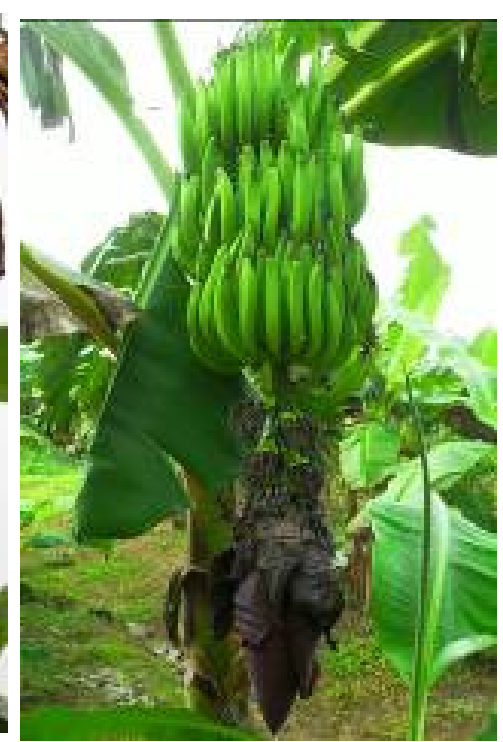

CNRA-PLA-18

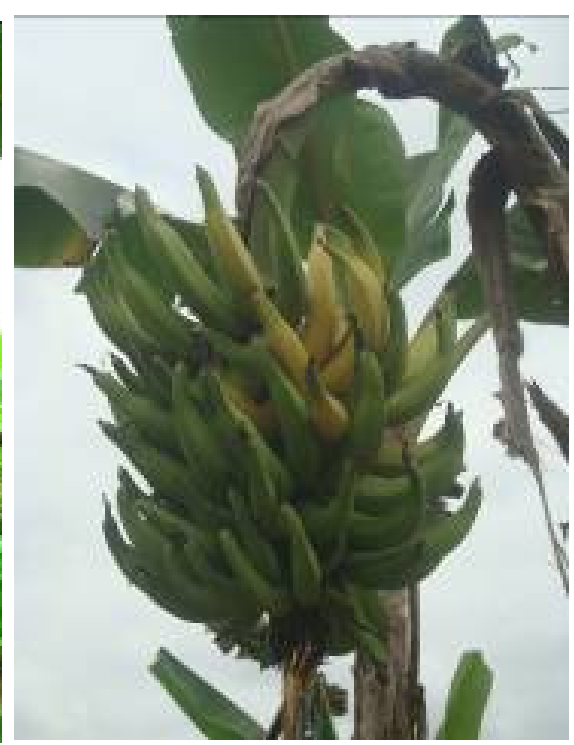

CNRA-PLA-9

Figure 4 : Les meilleures variétés locales de bananier plantain sélectionnées en Côte d'Ivoire

Les bananiers plantain ont été considérés comme précoce par les producteurs lorsqu'ils fleurissent dans les 12 mois après la plantation et tardif au-delà de ce laps de temps. Quatre types de plantain ont été identifiés dans les différentes zones de production en Côte d'Ivoire. Les types Faux corne sont les plus cultivés pour des raisons économiques à cause de la taille de leurs fruits plus gros et plus longs que ceux du type French. En effet, les fruits de grande taille sont beaucoup appréciés sur les marchés et se vendent mieux (Nguessan et al., 1993). L'évaluation et la sélection participative des variétés a permis d'impliquer les producteurs dans le choix des variétés en fonction de leur préférence. Les producteurs qui ont participé aux essais avaient l'expérience de la production bananière et s'y intéressaient énormément dans leur zone. Les performances agronomiques des variétés évaluées ont été stables dans les différentes zones agroécologiques. Leurs performances n'ont pas été influencées par les conditions climatiques des localités. Des résultats similaires ont été enregistrés lors de la 
caractérisation agro-morphologique à la station expérimentale d'Azaguié. Ainsi, en appliquant de bonnes pratiques culturales, les producteurs seraient assurés d'obtenir de bons rendements avec ces variétés. Les variétés CNRA-PLA-3, CNRA-PLA-4, CNRA-PLA-15, CNRA-PLA-7, CNRA-PLA-18 et CNRA-PLA-9 ont conservé davantage de feuilles fonctionnelles à la floraison que les autres variétés, ce qui pourrait être l'une des explications de leur rendement plus élevé. En effet, un nombre de feuilles fonctionnelles à la floraison supérieur ou égale à 8 feuilles garantie un bon rendement (Lassoudière, 2007). Les variétés évaluées ont exprimé les symptômes de la cercosporiose noire. Nos résultats confirment ceux de Vuylsteke et al. (1993a). Les auteurs avaient montré que tous les variétés de bananiers plantain et certaines variétés de bananiers d'Afrique de l'Est sont sensibles à cette affection fongique (Vuylsteke et al. 1993a). Le rang de la plus jeune feuille nécrosée a été plus important dans la localité de Katiola que dans

\section{CONCLUSION}

La Côte d'Ivoire renferme une forte diversité variétale de banane plantain centrée dans la partie Est du pays où plusieurs variétés cultivées ont été identifiées. L'exploitation et la valorisation de cette biodiversité a permis d'identifier 6 variétés (CNRA-PLA-3, CNRA-PLA4, CNRA-PLA-15, CNRA-PLA-7, CNRA-PLA-18 et CNRA-PLA-9) à haut potentialité de rendement avec des poids de régime variant de 25 à $29 \mathrm{~kg}$ en milieu paysan et

\section{REMERCIEMENTS}

Cette recherche a été supportée par le Programme de Productivité Agricole en Afrique de l'Ouest (PPAAO/WAAPP) financé par la banque mondiale. Nous remercions, les agents d'ANADER (Agence Nationale

\section{REFERENCES BIBLIOGRAPHIQUES}

Anonyme, 2010. Etude de faisabilité du projet de production de banane plantain de contre saison dans le sud forestier de la Côte d'Ivoire. Rapport final, FIRCA, BNETD, CNRA. 108 pp.

Frison EA, Gold CS, Karamura EB, 1998. Mobilizing IPM for sustainable banana production in Africa. Inibap. Proceedings of a workshop on banana IPM held in Nelspruit, South Africa 23-28 November 1998.

IPGRI/INIBAP, CIRAD, 1996. Descriptors for Banana (Musa spp.). IPGRI, Rome, Italy/INIBAP, Montpellier, France/CIRAD, France. 55 pp. Available from: http://www. celle de Bouaflé. Cette différence pourrait s'expliquer par les conditions climatiques et notamment par la température qui n'est pas la même dans les deux régions. La région de katiola au Nord de la Côte d'Ivoire est plus sèche avec des températures élevées que la région de Bouaflé dans la partie forestière, plus humide avec des températures plus basses. En effet, la cercosporiose noire se développe plus rapidement en condition climatique humide. Des résultats similaires ont été mis en évidence par Traoré (2008). L'auteur a observé des symptômes plus sévères de la maladie et un rang de la plus jeune feuille nécrosée plus faible en condition de pluie qu'en saison sèche. Le choix des participants à la sélection participative a porté sur les variétés 6 variétés CNRA-PLA-3, CNRA-PLA-4, CNRA-PLA-15, CNRA-PLA7, CNRA-PLA-18 et CNRA-PLA-9 à cause de leur poids de régime élevé (Figure 4). Les 3 variétés CNRA-PLA-3, CNRA-PLA-4, CNRA-PLA-15, ayant de gros et longs doigts ont été les plus préférées.

moins sensible à la cercosporiose noire. Ces variétés locales sélectionnées par les producteurs, ont des cycles de production courts et sont déjà adaptées aux différents mets locaux. Leur vulgarisation en milieu paysan contribuera à l'amélioration de la productivité de la banane plantain en Côte d'Ivoire et permettra aux producteurs d'accroître leur revenu.

d'Appui au Développement) des zones Est, CentreOuest, Sud-Ouest et Ouest et les populations des villages visités qui nous ont accueillis avec beaucoup de gentillesse.

bioversityinternational.org/fileadmin/bioversity/ publications/pdfs/326.pdf. Date accessed: 2 December 2008.

Kouassi KS, 2006. Rôle des ressources génétiques dans l'essor du secteur bananier plantain en Côte d'Ivoire. Rapport d'activité 2006. Document CNRA.

Lassoudière A., 2007. Le bananier et sa culture. Montpellier, Quae. 383 pp.

Nguessan A, Yao N, Kehe M, 1993. La culture du bananier en Côte d'Ivoire. Fruit 48: 133-143. 
Nkendah R, 2001. Collecte et analyse des données secondaires sur la filière bananes et plantains au Gabon. Rapport technique de mission. $25 \mathrm{pp}$.

Temple L, Bikoï A, Tallec F, 2001. Collecte et analyse des données secondaires sur les productions bananières au Cameroun. Rapport Final. Document CRBP N ${ }^{\circ}$ 230/CRBP/2001. 30 pp.

Tetang J, 2013. Bananier plantain: Production des semences saines par la technique PIF, Conduite d'une exploitation de plantain et Les variétés les plus productives. La voix du paysan $260: 6-14$.

Traore S, Kobenan K, Kouassi S, Gnonhouri GP, 2009. Systèmes de culture du bananier plantain et méthodes de lutte contre les parasites et ravageurs en milieu paysan en Côte d'Ivoire. Journal of Applied Biosciences 19: 1094-1101.

Traoré S, 2008. Contribution à l'étude de comportement d'hybrides de bananiers de dessert et de bananiers plantain (Musa sp.) vis-à-vis des parasites foliaires (mycosphaerella spp., cladosporium musae) et racinaires (zythia sp., radopholus similis, pratylenchus coffeae) en Côte d'lvoire. Thèse de Doctorat es Sciences, Physiologie Végétale, UNIVERSITE DE COCODY ABIDJAN (Côte d'Ivoire), $195 \mathrm{pp}$.

Vuylsteke D, Swennen R, Ortiz R, 1993a. Registration of 14 improved tropical Musa plantain hybrids with black Sigatoka resistance. HortScience 28: 957959. 